\title{
JASMINE data analysis
}

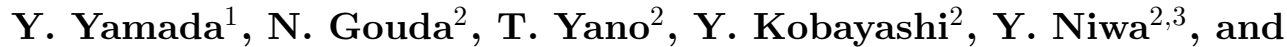 JASMINE Working group}

\author{
${ }^{1}$ Department of Physics, Kyoto University, \\ Oiwake-cho Kita-Shirakawa Kyoto 606-8502 JAPAN \\ email:yamada@amesh.org \\ ${ }^{2}$ National Astronomical Observatory of JAPAN, \\ National Institutes of Natural Sciences, Mitaka, Tokyo, 181-8588, JAPAN \\ email:naoteru.gouda@nao.ac.jp, yuki@merope.mtk.nao.ac.jp,t.yano@nao.ac.jp \\ ${ }^{3}$ Graduate School of Human and Environment Study, \\ Kyoto University Kyoto 606-8501, JAPAN \\ email:kazin.niwa@nao.ac.jp
}

\begin{abstract}
Japan Astrometry Satellite Mission for Infrared Exploration (JASMINE) aims to construct a map of the Galactic bulge with a 10 mas accuracy. We use z-band CCD or K-band array detector to avoid dust absorption, and observe about $10 \times 20$ degrees area around the Galactic bulge region.

In this poster, we show the observation strategy, reduction scheme, and error budget. We also show the basic design of the software for the end-to-end simulation of JASMINE, named JASMINE Simulator.
\end{abstract}

Keywords. methods: data analysis

\section{Introduction}

JASMINE will observe bulge stars with a single telescope and create accurate map using the block-adjustment algorithm (see Eichhorn \& Clary(1974) and Zacharias et al. (1992)). Each observation corresponds to a "Small Frame". By adjusting several thousand Small Frames from about 10-hrs of observations, we obtain a "Large Frame". By adjusting about 2000 Large Frames, we can get astrometric parameters of bulge stars with high accuracy. In the plate mapping method, exposure time can be adjusted accordingly for each FoV in order to minimize the errors within the limits of hardware and mission's life. JASMINE will observe about 1 million bulge stars with an accuracy of $\sigma / \pi<0.1$.

\section{Instrument and observation strategy overview}

The JASMINE telescope is a three mirror system. The diameter of the primary mirror is $75 \mathrm{~cm}$, and the focal length is $22.5 \mathrm{~m}$ in z-band option or $13.5 \mathrm{~m}$ in K-band option. Observations are performed within $20^{\circ} \times 10^{\circ}$ region using $0.7^{\circ} \times 0.7^{\circ}$ FoVs. Because the stellar density is very high, we can apply block adjustment method and the beam combiner which is used in HIPPARCOS and Gaia is not needed. Our satellite has single telescope without beam combiner and like the ordinary space telescope. The telescope has very long focal length because the PSF size should be larger than several pixels for centroiding. There are 4 plane mirrors to fold the long optical path.

The constructing CCD detector which is sensitive to z-band $(0.9 \mu \mathrm{m})$ is almost completed in collaboration of Subaru telescope and Japanese company (see Kamata et al. (2006)). Other candidate of detector is $\mathrm{HgCdTe} \mathrm{K}$-Band array detector. 
In the galactic bulge, we can adjust each frames with very high accuracy from the simple statistical considerations because density of stars are very high as the block adjustment algorithms for astrometry. About 5000 stars are observed within each "Small frame", we may expect that the accuracy of determining plate parameters is about $30 \sim 50$ times better than that of each stellar observation in the statistical sense.

Diffraction limit of our telescope will be about 100 milli arc-sec. From the picture of FoV, relative distance between stars in the FoV are calculated about 1/200 diffraction limit accuracy. By adjusting $10^{7} \mathrm{FoVs}$, we can solve the astrometric parameters of 10 $\mu$ as accuracy.

In the process of spacial connection of each "Small Frame" to construct "Large Frame", we estimate both the location and size of each "Small Frames". Higher order mapping parameters such as distortion cannot be estimated within the limitation of aperture size. So, optics deformation should be less than that the distortion and other higher order mapping parameters changes are not essential for position determination. By using Laser Interferometer technique which is developed in the gravitational wave detection, deformations of instruments are monitored.

JASMINE mission areas are very close to the ecliptic, errors along the ecliptic longitude direction is important and may affect the precision of astrometric parameter estimation. By optimizing reduction processes and appropriate choice of coordinates, accumulating errors of ecliptic longitude direction by adjustment processes can be $0.1 \sigma \sim 0.2 \sigma$ in "Large frame" , where $\sigma$ is the accuracy of centroiding in each star. So, it is very important to prevent or monitor the deformation of mirror and telescope frames.

It is considered that very long time (order of an year) calibration of scale is needed in the estimation of astrometric parameters. Because the calibration of the hardware in such a long time is impossible, HIPPARCOS satellite uses beam combiner. But it is needed that only linear and periodic with one year period variation may cause the degeneracy to astrometric parameters. So we only need to estimate above two modes in enough accuracy. Any other variation modes can be considered to be random errors and which may not be a bias in the estimation of astrometric parameters. For final calibration, we use QSOs. There are about 40 QSO candidates in our mission area, and 3 to 5 QSOs will be able to be observed by JASMINE.

\section{Constructing end-to-end simulation tool}

We are now constructing end-to-end simulation software. The basic design and implementation is completed. The software is coded by using event-driven architecture. The simulator is applied for developing and checking algorithms for attitude control in Nano-JASMINE satellite.

\section{References}

Eichhorn, H., \& Clary, W. G. 1974, MNRAS, 166, 425

Kamata, Y., Miyazaki, S., Nakaya, H., Tsuru, T. G., Takagi, S., Tsunemi, H., Miyata, E., Muramatsu, M., Suzuki, H., \& Miyaguchi, K. 2006, High Energy, Optical, and Infrared Detectors for Astronomy II. Edited by Dorn, David A.; Holland, Andrew D.. Proceedings of the SPIE, , 6276, 62761U.

Zacharias, N., de Vegt, C., Nicholson, W., \& Penston, M. J. CPC2 - the Second Cape Photographic Catalog II. Conventional plate adjustment and catalog construction. 1992 A\&A 254,397 